\title{
\#DíalnternacionaldelPuebloGitano: una mirada a la realidad educativa de la etnia gitana a través de Twitter
}

\section{\#DíalnternacionaldeIPuebloGitano: a look at the educational reality of the gypsy through Twitter}

\author{
Patricia Motos Sellés ${ }^{1}$, Ángel Ignacio Aguilar Cuesta ${ }^{2}$ \\ ${ }^{1}$ Universidad Internacional de Valencia, España (pmotos@universidadviu.com) \\ ${ }^{2}$ Universidad Internacional de Valencia, España (angelignacio.aguilar@universidadviu.com)
}

Recibido el 18 de junio de 2018; revisado el 24 de septiembre de 2018; aceptado el 24 de septiembre de 2018; publicado el 1 de diciembre de 2018

\section{RESUMEN:}

El presente estudio trata de identificar la actividad y demandas educativas asociadas con la etnia gitana dentro de España a través de la red social Twitter, con el fin de determinar cuáles son los diversos dictámenes sobre esta cuestión. Para ello, hemos empleado una metodología híbrida basada, por un lado, en el análisis cuantitativo de los tweets recogidos gracias al uso de la minería de datos y estructura de redes a través de la API de Twitter. Por otro, una metodología cualitativa debido al estudio de todos los tweets extraídos con el hashtag \#DíaInternacionaldelPuebloGitano, gracias al cual hemos determinado las demandas y opiniones, así como la catalogación emocional de dichos textos. El análisis de los 726 tweets en lenguas oficiales del Reino de España, siendo este elemento territoriallingüístico un delimitador utilizado para comprender la opinión pública en un espacio geográfico concreto.

El análisis de los datos se ha realizado empleando el programa SPSS 21 con el cual hemos podido determinar que el número de tweets emitidos por los usuarios fue de 726, cuya propagación aumentó debido a los retweets y favoritos hasta un total de 2061 en el día 8 de abril. La franja horaria de mayor actividad fue de 12:00-17:59, con un total de 959.

Finalmente, hemos podido determinar como la actividad en la red social tiene grandes similitudes con los estudios hasta la fecha sobre la etnia gitana en relación con la educación. A saber: las protestas y quejas por la desigualdad y la intolerancia, la existencia y funcionamiento de centros escolares donde existe un alto número de alumnado gitano. Por el contrario, también encontramos similitudes, como el incremento lento de alumnado de etnia gitana con mayores estudios, y que son vistos tanto en redes sociales como dentro de la comunidad como ejemplos a seguir.

\section{PALABRAS CLAVE: EDUCACIÓN, NECESIDADES DEL ALUMNADO, REDES SOCIALES, ESPAÑA.}

\begin{abstract}
:
The current paper tries to identify the activity and educational demands associated with the ROMA inside Spain across Twitter, in order determine which are the diverse opinions on this question. For it, we have used a hybrid based methodology on the one hand, in the quantitative analysis of the tweets gathered thanks to the use of the data mining and structure of networks across Twitter's API. For other one, a qualitative methodology due to the study of all the tweets extracted with the hashtag \#DíaInternacionaldelPuebloGitano, thanks to that we have determined the demands and opinions as well as, the emotional cataloguing of the above mentioned texts. The analysis of the 726 tweets in official languages of the Kingdom of Spain, being this territorial - linguistic element a delimitator used to include the public opinion in a geographical concrete space.

The analysis of the information has been realized using the software SPSS 21 with which we could
\end{abstract}


have determined that the number of tweets issued by the users was of 726, whose spread increased due to the retweets and favorites up to a total of 2061 in April 8. The busiest time was of 12:00-17:59 with a total of 959.

Finally, we could have determined as the activity in the social network, it has great similarities with the studies up to the date on the ROMA in relation with the education. Thus, the protests and complaints for the inequality and the intolerance, the existence and functioning of school centers where there exists a high number of ROMA student body. Conversely, also we find similarities, as the slow increase, of student body of ROMA with major studies, and that are seen so much in social networks like inside the community, as examples to continuing.

\section{KEYWORDS: EDUCATION, CHILDHOOD NEEDS, SOCIAL NETWORKS, SPAIN.}

\section{INTRODUCCIÓN}

El aparición y desarrollo de la Tercera Revolución Industrial (Rifkin, 2011) y la inminente llegada de la cuarta (Schwab, 2016), ha provocado que la tecnología modifique las formas de relación humana, siendo las redes sociales un claro y actual ejemplo. Estas nuevas formas de conectividad con las personas o colectivos (Bennett y Seberger, 2013) que se expresan mediante estas redes están suponiendo una fuente de estudio continua, gracias a la estructuración y capacidad para extraer datos de forma automática de todo lo que sucede $\mathrm{y}$ ha sucedido en redes sociales.

Este elemento ha provocado que Twitter, microblogging, creado en 2006, sea objeto de estudio con el fin de determinar patrones, actitudes, creencias, comportamientos, etc., de un colectivo o individuo a través de los datos que aporta el uso de esta herramienta (Adnan, Leak y Longleya, 2014; Jackson y Wang, 2013), pero, sin duda, uno de los elementos más interesantes son los estudios comparados (Tur, Marín y Carpenter, 2017) con otros espacios geográficos, meta a la que nos proponemos llegar en un futuro próximo.

Si nos centramos en España, cabe destacar cómo hemos experimentado en la última década una evolución sin precedentes en el uso de las redes (Garmendia et al., 2011). Así pues, el 86\% de las personas entre los 16 y 65 años de edad son usuarios de las redes sociales (Iab Studio, 2018), destacando los jóvenes por encima del resto (Sánchez Rodríguez, Ruiz Palmero, y Sánchez Rivas, 2015).

Por otra parte, cabe hacer mención a los hechos que nos han traído hasta el estudio de la etnia gitana a través del hashtag \#DíaInternacionaldelPuebloGitano, el cual nos ha permitido analizar en profundidad lo dicho sobre la comunidad romaní en Twitter. Por un lado, y sin ánimo de ser extensos en la explicación, debemos tener en cuenta que, en relación con el ámbito educativo, desde posturas asimilacionistas se considera este elemento como una asimilación de culturas y valores distintos a los romaní, por lo que pueden generar rechazo (Garreta, 1998). Por otro lado, los diversos planes puestos en marcha para incrementar la tasa de población gitana con estudios post-obligatorios -véanse los objetivos recogidos dentro de la Estrategia Inclusión Gitana 2012-2020-, van dirigidos al aumento de titulados en las diferentes etapas educativas y, sobre todo, tal y como puso de manifiesto la Fundación Secretariado Gitano, evitar que el $64 \%$ de alumnado romaní de entre 16-24 años no concluya sus estudios. Además, este último elemento afecta de lleno según el sexo, dado que a los 16 años las chicas escolarizadas -un $48 \%$ - son menor en número que los chicos $-63,4 \%-$.

Así pues, debemos tener en cuenta la relevancia educativa como mecanismo de equidad e integración. Pero esta idea se contrapone si analizamos los diversos estereotipos de la etnia gitana a través de los medios de comunicación, denunciado dentro del Informe Anual FSG "Discriminación y Comunidad Gitana" (Fundación Secretariado Gitano, 2017), según el cual, durante el año 2016, hubo un total de 202 casos de discriminación, de los cuales el 5,45\% fueron de ámbito educativo y todos de discriminación directa afectando a menores.

Tabla 1. Número de los casos de discriminación hacia la etnia gitana en 2016 por ámbitos

\begin{tabular}{ccc}
\hline Ámbitos & Número & Porcentaje \\
\hline $\begin{array}{c}\text { Medios de } \\
\text { Comunicación e } \\
\text { Internet }\end{array}$ & 68 & 33,66 \\
\hline Empleo & 44 & 21,78 \\
\hline Acceso a bienes & 28 & 13,86 \\
\hline Otros "Discurso de & 24 & 11,88 \\
odio" & 12 & 5,94 \\
\hline Vivienda & 11 & 5,45 \\
\hline Educación & 10 & 4,95 \\
\hline Servicios policiales & 5 & 2,48 \\
\hline Salud & 202 & 100 \\
\hline Total & &
\end{tabular}

Elaboración propia. Fuente: Informe anual Fundación Secretariado Gitano "Discriminación y Comunidad Gitana”, 2017. 
Si nos centramos ahora en los estudios que han analizado las redes sociales desde un punto de vista educativo, y más concretamente empleando Twitter (Moreno, Navas, y Ortega, 2017; Valdiviezo, Viteri y Carrera, 2017), nos damos cuenta que existe una multitud de trabajos que giran bien sobre el uso de Twitter como herramienta educativa y sus derivadas formas de enseñanza-aprendizaje (De-Paoli y Larooy, 2015; Rattray y Lavery, 2015), o bien sobre el análisis de los datos y publicaciones emitidos dentro de la red social (Davis, 2015; West, Moore y Barry, 2015).

Teniendo todo ello en cuenta, debemos advertir que los objetivos concretos de nuestro estudio pasan por:

1. Determinar la actividad e impacto en Twitter del hashtag \#DíaInternacionaldelPuebloGitano.

2. Analizar de manera cuantitativa y cualitativa el contenido de los tweets emitidos por los usuarios, centrándonos en aquellos que hablan de educación.

3. Determinar y comparar las afirmaciones emitidas en Twitter con los informes y estudios sobre la educación en relación con la etnia gitana, comprobando su similitud o diferencia.

\section{DISEÑO Y METODOLOGÍA}

Como hemos afirmado con anterioridad, la metodología híbrida nos ha llevado a un desarrollo del estudio por etapas. Primeramente, la creación de un script para que descargase de la API Rest de Twitter todos los tweets con el hashtag \#DíaInternacionaldelPuebloGitano (y también, \#DiaInternacionaldelPuebloGitano dado que a veces los usuarios no acentúan el hashtag), a partir del cual recopilar toda la información emitida en España durante el día 8 de abril del 2018.

Figura 1. Elementos del script creado para la extracción de tweets

1 def process_results (results):

id_list $=$ [tweet.id for tweet in results]

data_set $=$ pd.DataFrame $\left(i d \_l i s t\right.$, columns $\left.=[" i d "]\right)$

data_set ["text"] = [tweet.text for tweet in results] data_set ["created_at"] = [tweet.created at for tweet i data_set ["retweet_count"] = [tweet. retweet_count for $t$ data_set ["favorite_count"] = [ tweet.favorite_count for data_set["source"] $=$ [tweet. source for tweet in result data_set["user_id"] = [tweet.author.id for tweet in re data_set["user_screen name"] = [tweet.author.screen na data_set ["user_name"] = [tweet. author, name for tweet i data_set ["user_created_at"] = [tweet.author. created at data_set ["user coscription"] = [tweet.author.descripti data_set ["user_dollowers count"] = [tweet.author follo data_set ["user_friends_count"] = [tweet. author. friends

Elaboración propia. Fuente: Spyder (Anaconda)
El total de tweets ascendió a 726, cuya difusión debido a los retweets y favoritos aumentó a 2061, aunque si tenemos en cuenta los días anteriores y posteriores al 8 de abril, su número se incrementa hasta los 2594 tweets. De ellos, pudimos obtener como categoría de análisis cuantitativo, tanto por el contenido emitido, los hashtag, el número de retweets y favoritos, así como las menciones, contabilización de las palabras más usadas dentro de los tweets, como cualitativo por la contrastación y encuadre de cada uno de los 726 tweets.

A partir de aquí, se emplearon dos tipos de diferenciaciones: la primera para determinar la característica del mensaje emitido hacia la etnia gitana y/o el Día Internacional del Pueblo Gitano, entre las que están: apoyo, desagrado, formativo, informativo, orgullo, participativo, y reivindicativo (Sarlan, Nadam y Basri, 2014). La segunda diferenciación dentro de nuestro análisis sirvió para dividir aquellos tweets de carácter educativo, y los que no lo son, con el fin de determinar las similitudes y diferencias con los análisis y estudios sobre la educación romaní en España.

\section{RESULTADOS}

El análisis de los resultados obtenidos nos ha permitido ver con claridad tanto la propagación del hashtag \#DíaInternacionaldelPuebloGitano, como las interacciones de los usuarios a nivel estatal. En este caso, tal y como afirmábamos antes, la propagación debido a los retweets y favoritos ascendían a un total de 2061 durante el 8 de abril de 2018, mientras que, si tenemos en cuenta las fechas anteriores y posteriores, vemos también actividad, pero de una forma más lenta.

Figura 2. Número de tweets capturados con el hashtag \#DíaInternacionaldelPuebloGitano del 5 al 9 de junio de 2018

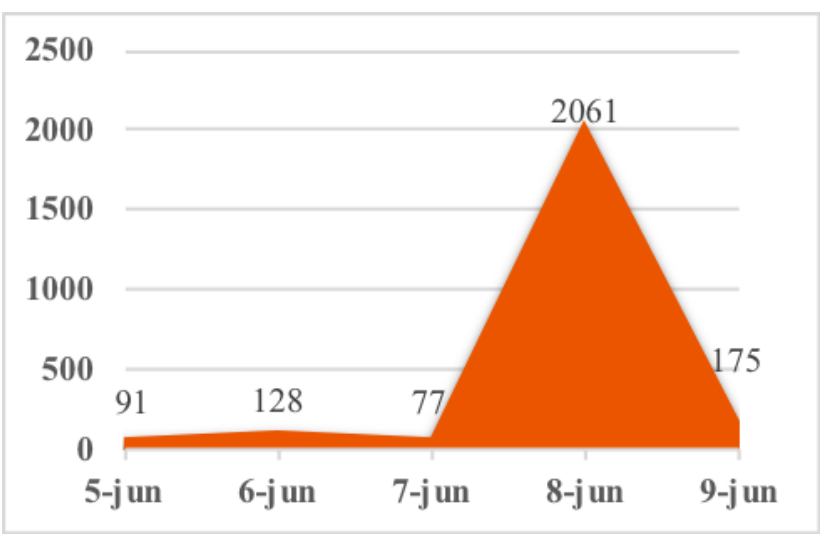

Elaboración propia. Fuente: Tweets extraídos. 
Si nos adentramos en el análisis pormenorizado de la actividad en las franjas horarias del día 8, observamos como entre las 00:00 y 05:59 únicamente se emitieron 5 tweets, desde las 06:00 hasta las 11:59 fueron publicados 432 tweets, la franja horaria con mayor actividad se produjo desde las 12:00 hasta las 17:59 con 959 tweets publicados; finalmente, en la última franja horaria del día bajó el número de tweets, un total de 665 .

Tabla 2. Actividad horaria del hashtag \#DíaInternacionaldelPuebloGitano el 8 de abril de 2018.

\begin{tabular}{ccc}
\hline Horario & $\mathrm{N}^{\mathrm{o}}$ de tweets & $\%$ \\
\hline $00: 00-05: 59$ & 5 & 0,24 \\
\hline $06: 00-11: 59$ & 432 & 20,96 \\
\hline $12: 00-17: 59$ & 959 & 46,53 \\
\hline $18: 00-23: 59$ & 665 & 32,27 \\
\hline Día completo & 2061 & 100
\end{tabular}

Elaboración propia. Fuente: tweets publicados

El primero de ellos, fue publicado por el Ministerio de Educación, Cultura y Deporte del Gobierno de España: @MEpublicaciones, a las 00:35. No obstante, hubo un retweet de @ csdiazguerra a las 00:15 del tweet emitido por Juan Trinidad Martos @ juantri73 el día 7 de abril.

Figura 3. Primer tweet publicado por un usuario con el hashtag \#DíaInternacionaldelPuebloGitano el 8 de abril.

Publicaciones MECD @MEpublicaciones · 8 abr.

\#DíalnternacionalDelPuebloGitano Historia de vida de $\mathbf{5 0}$ estudiantes gitanos y gitanas bit.ly/1V6xyL4
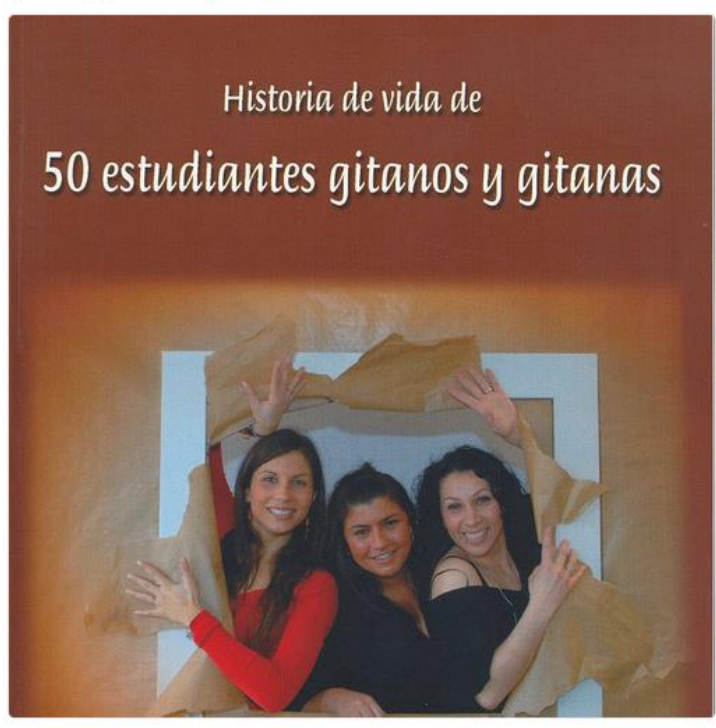

Q $\quad$ ᄂ $1 \quad \bigcirc_{2} \quad \square$

Fuente: @MEpublicaciones en Twitter (https://twitter.com/MEpublicaciones/status/98299649805 $\underline{1813377)}$

Ahora bien, una vez detallado el alcance del hashtag a lo largo de la red social, pasamos al análisis pormenorizado de los tweets emitidos de manera directa, es decir, dejando de lado los retweets y favoritos. De esta forma, nos encontramos con un total de 726 tweets que han sido los estudiados al detalle.

Todos ellos, se dividieron en 7 tipologías según las características del contenido del tweet:

1. Apoyo: mensajes de carácter positivo hacia la etnia gitana.

2. Desagrado: mensajes críticos en tono despectivo por el trato social dado a la etnia gitana.

3. Formativo: mensajes cuyo contenido principal es formar a los usuarios que lo leen sobre la cultura, tradiciones, etc. del pueblo gitano.

4. Informativo: mensajes con información del día 8 de abril y la etnia gitana.

5. Orgullo: mensajes de exaltación sentimental de la etnia gitana.

6. Participativo: mensajes que hacen un llamamiento a la participación social en las actividades propuestas para conmemorar el día internacional de la etnia gitana.

Figura 4. Ejemplo de tweet participativo emitido el 8 de abril por@aytorincon.

Ayto Rincón Victoria @aytorincon - 8 abr.

¡Buenos días y \#felizdomingo! Hoy celebramos el

\#DíalnternacionaldelPuebloGitano it y en \#RincónDeLaVictoria lo

celebramos con una jornada muy especial a las $12 \mathrm{~h}$ de la mañana en la Plaza Al-Ándalus. Por una igualdad real y sin discriminaciones

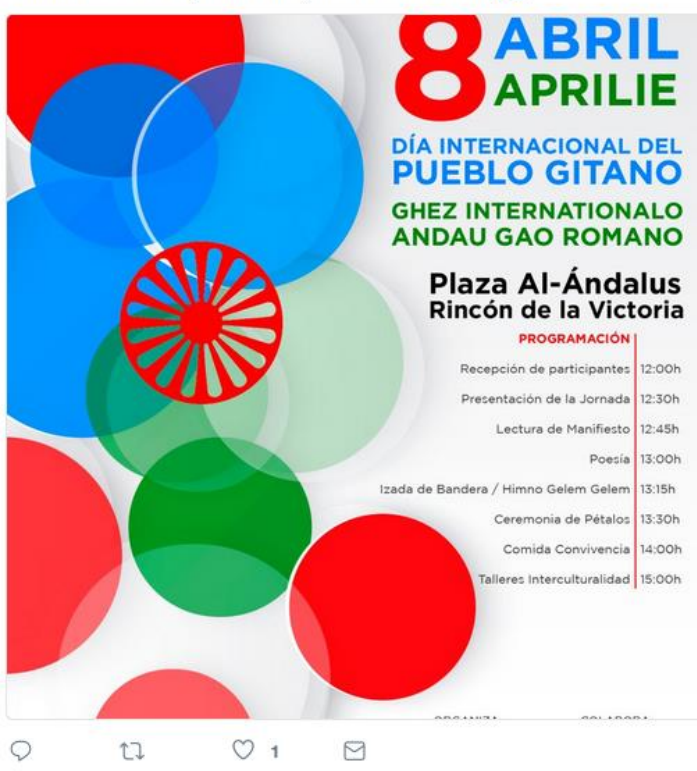

Fuente: @aytorincon en Twitter

(https://twitter.com/aytorincon/status/9828833055100477 44)

7. Reivindicativo: mensajes críticos cuyo finalidad es la de cambiar o denunciar la realidad social de la etnia gitana en España. 
Tabla 3. Número de tweets emitidos según su tipología

\begin{tabular}{ccc}
\hline Tipología & $\mathrm{N}^{\mathbf{o}}$ de tweets & $\%$ \\
\hline Apoyo & 249 & 34,30 \\
\hline Desagrado & 2 & 0,28 \\
\hline Formativo & 36 & 4,96 \\
\hline Informativo & 279 & 38,43 \\
\hline Orgullo & 14 & 1,93 \\
\hline Participativo & 77 & 10,61 \\
\hline Reivindicativo & 69 & 9,50 \\
\hline Total & 726 & 100 \\
\hline
\end{tabular}

Elaboración propia. Fuente: Tweets extraídos

Entrando ahora en la parte fundamental del estudio, el impacto de esos tweets en el entorno educativo, comenzamos por el número de tweets relacionados con dicha temática. Los clasificados como educativos se entienden como aquellos tweets cuyo contenido tiene un carácter didácticopedagógico, ya sea en un entorno de aprendizaje de carácter formal o informal.

De este modo, del total de 726 tweets, 429 (59\%) no son de carácter educativo, mientras que el resto, 297 (41\%), sí han sido catalogados como tales.

Figura 5. Gráfica del número de tweets educativos y no educativos con el hashtag

\#DíaInternacionaldelPuebloGitano

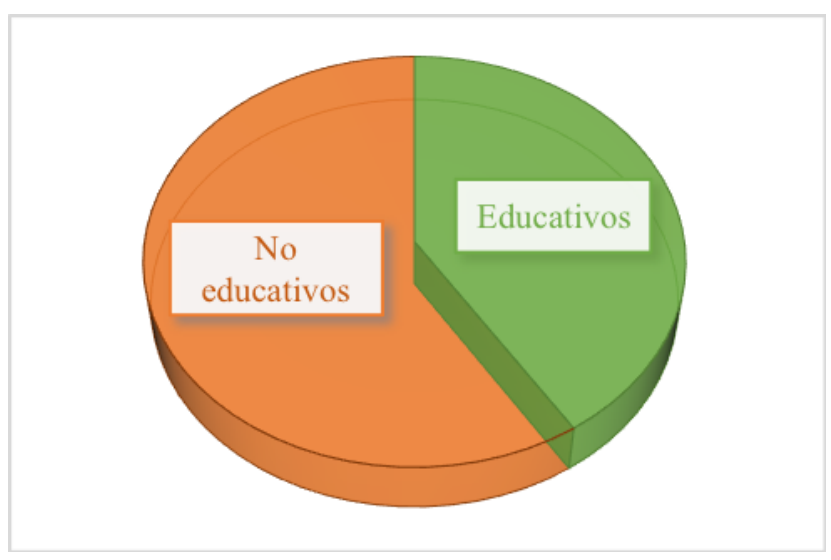

Elaboración propia. Fuente: Tweets extraídos

Los tweets emitidos van, desde el punto de vista educativo, hacia la reivindicación y el apoyo por la una participación inclusiva de la etnia gitana en la educación y la eliminación del tópico y las barreras invisibles para alcanzarlos.

En este sentido, vemos estas reclamaciones a nivel general de la sociedad. Así, el Ministerio de Sanidad señalaba la necesidad de "eliminar las barreras invisibles, la intolerancia y los prejuicios contra el pueblo gitano".
Figura 6. Tweet emitido por el Ministerio de Sanidad del Gobierno de España.

Min. Sanidad (MSSSI) @sanidadgob · 8 abr.

Hoy 8 de abril, se celebra el \#DíalnternacionalDelPuebloGitano, en el que los gitanos de todo el mundo celebran la cultura gitana, y para concienciar a la sociedad de la eliminación de las barreras invisibles, la intolerancia y los prejuicios contra el pueblo \#gitano @Gitanos_org

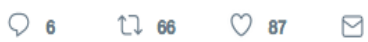

Fuente: @ sanidadgob en Twitter (https://twitter.com/sanidadgob/status/9829178544116121 $\underline{60)}$

También se expresaban así otros usuarios de la red como Maribel Mora Grande: @MaribelMoraG al exponer la importancia del 8 de abril para recordar que la etnia gitana continua siendo vista de manera estereotipada, con prejuicios y discriminación:

https://twitter.com/MaribelMoraG/status/982912939 509342208.

Otro de los ejemplos destacados son aquellos casos que ensalzan la figura de personas/entidades que trabajan por la educación fuera de los centros escolares. Entre ellos, Carlos Fernández destaca por usar el deporte, concretamente el boxeo, que ha permitido según la cuenta oficial de Castilla LaMancha Media: @CMM_es salvar "de la calle a 30 chicos y chicas": https://twitter.com/CMM_es/status/9829058962830 58177.

Centrándonos en el colectivo escolar, a nivel general encontramos demandas similares. Pero para ilustrarlo, hemos decidido hacerlo por obligatoriedad o no, de la enseñanza. Así, encontramos como @MareasXLaEP critica duramente al gobierno por no cumplir el artículo 14 de la Constitución Española y continuar con la agrupación de niños gitanos en aulas y centros escolares. Por ende, reivindican acabar con dicha segregación acompañándolo del hashtag \#NoQuieroUnaEscuelaSegregada

(https://twitter.com/MareasXLaEP/status/982933309 767462913). Todo ello, parte de una campaña promovida por Fundación Secretariado Gitano para visibilizar esta problemática a tenor de los datos obtenidos por de la encuesta realizada por la Agencia de los Derechos Fundamentales de la Unión Europea, donde se advierte que existe un $31 \%$ de niños gitanos que van a centros escolares con un número elevado de alumnado de etnia gitana ya en ellos (European Union Agency for Fundamental Rights, 2017).

En esta misma línea, el propio Defensor del Pueblo Español (@DefensorPuebloE) realizó una visita al CEIP Albolafia de Córdoba, centro de 
educación infantil y primaria donde la mayoría del alumnado es de etnia gitana (https://twitter.com/DefensorPuebloE/status/981871 247024893954). Esta realidad provocó la petición, con fecha del 5 de abril de 2018, al Ministerio de Educación, Cultura y Deporte de información "sobre los colegios en los que la población gitana es mayoritaria y sobre las medidas para evitar la concentración de estos niños y niñas en determinados centros escolares" (https://twitter.com/DefensorPuebloE/status/981877 662414245888).

Pasando a la etapa no obligatoria, es decir, Bachillerato y universidad, vemos como las demandas y mensajes son distintos. Se trata de ejemplificar los casos de éxito del alumnado de etnia gitana en dichos niveles educativos, convirtiéndose en modelos a imitar o seguir por el resto. El tweet más destacado, tanto por su difusión como por simbolizar claramente lo que hablamos, es el emitido por el Ministerio de Educación, Cultura, y Deporte: @MEpublicaciones, que recuperó un estudio sobre los "Gitanos en la Universidad" (Padilla Carmona, González Monteagudo y Soria Vílchez, 2017), publicado en Revista de Educación.

Es también llamativo los datos reflejados por el uso de los hashtags que acompañan al estudiado. Así, de los 264 recogidos, cuya contabilización ascendía a 2882 recopilados en todos los tweets, los más empleados obviamente fueron \#DiaInternacionaldelPuebloGitano que supone un $57,22 \%$ del total. Seguidamente aparecían \#8deabril (7,01\%), \#Andalucía \#diainternacionaldelpoblegitano $(1,87 \%)$.

$\mathrm{Si}$ nos detenemos en los mensajes que han recibido un mayor número de retweet, nos encontramos que el valor más alto se halla en 137, seguido de $92 \mathrm{y}$, posteriormente, varios oscilan desde los 91 hasta los 77 retweets.

Tabla 4. Valores más altos de retweets alcanzados con el hashtag \#DíaInterancionaldelPuebloGitano

\begin{tabular}{|c|c|c|}
\hline Usuario & Número & URL \\
\hline PSOE & 137 & $\frac{\text { https://twitter.com/PSOE/status/9 }}{83068082141908993}$ \\
\hline $\begin{array}{l}\text { mjsanch } \\
\text { ezrubio }\end{array}$ & 92 & $\begin{array}{c}\text { https://twitter.com/mjsanchezrubi } \\
\text { o/status/982896906987765761 }\end{array}$ \\
\hline $\begin{array}{l}\text { rakeld12 } \\
341234\end{array}$ & 91 & $\begin{array}{l}\text { https://twitter.com/rakeld123412 } \\
\text { 34/status/982922402224136195 }\end{array}$ \\
\hline $\begin{array}{l}\text { pepecam } \\
\text { postruji }\end{array}$ & 85 & $\begin{array}{l}\text { https://twitter.com/pepecampostr } \\
\text { uji/status/982952833619292160 }\end{array}$ \\
\hline $\begin{array}{l}\text { gar_man } \\
\text { chon }\end{array}$ & 77 & $\begin{array}{l}\text { https://twitter.com/gar manchon/ } \\
\text { status/982912023024619520 }\end{array}$ \\
\hline
\end{tabular}

Elaboración propia. Fuente: Tweets extraídos
Analizando los números de favoritos obtenidos encontramos una estructura similar a la anterior.

Tabla 5. Valores más altos de favoritos alcanzados con el hashtag \#DíaInterancionaldelPuebloGitano

\begin{tabular}{|c|c|c|}
\hline Usuario & Número & URL \\
\hline PSOE & 137 & $\frac{\text { https://twitter.com/PSOE/status/9 }}{83068082141908993}$ \\
\hline $\begin{array}{l}\text { pepecam } \\
\text { postruji }\end{array}$ & 132 & $\begin{array}{l}\text { https://twitter.com/pepecampostr } \\
\text { uji/status/982952833619292160 }\end{array}$ \\
\hline $\begin{array}{c}\text { rakeld12 } \\
341234\end{array}$ & 119 & $\begin{array}{l}\text { https://twitter.com/rakeld123412 } \\
\text { 34/status/982922402224136195 }\end{array}$ \\
\hline $\begin{array}{l}\text { mjsanch } \\
\text { ezrubio }\end{array}$ & 109 & $\frac{\text { https://twitter.com/mjsanchezrubi }}{\text { o/status/982896906987765761 }}$ \\
\hline $\begin{array}{l}\text { Vicen01 } \\
03\end{array}$ & 83 & $\frac{\text { https://twitter.com/Vicen0103/sta }}{\text { tus/982877583267303424 }}$ \\
\hline
\end{tabular}

Elaboración propia. Fuente: Tweets extraídos

\section{DISCUSIÓN}

El análisis de Twitter nos ha permitido conocer la influencia y actividad del hashtag \#DíaInternacionaldelPuebloGitano. Nos indica las opiniones y valoraciones de los usuarios, tanto por el contenido emitido desde el punto de vista cualitativo como por la cantidad desde el cuantitativo.

Esas valoraciones, encuadradas según la característica del mensaje, muestran una mayoría notable de aquellos cuya finalidad es informativa y de apoyo, con el 72,73\%. No obstante, lo paradójico en este caso lo encontramos en el número de tweets con carácter desagradable, y en algún caso insultante, hacia la etnia gitana, que supone $0,28 \%$. ¿Por qué? Si recordamos los datos aportados al comienzo de nuestro estudio sobre los casos de discriminación, presentados por la Fundación Secretariado Gitano durante el 2016, observamos totalmente lo contrario, ya que el $33,66 \%$ de esa discriminación provenían del ámbito de los medios de comunicación e Internet. Se trataría de algo frecuente en las redes sociales, donde normalmente los ataques directos y malsonantes provienen de cuentas con perfiles falsos o no verificados (Ruiz Palmero, Sánchez Rodríguez, y Trujillo Torres, 2016; Vera y Dennys, 2017). Aún así, el rechazo y estigmatización que genera posteriormente las afirmaciones como esas, hacen que muchos usuarios no las emitan de forma pública, es decir, se silencia, pero no se intenta mejorar la empatía hacía dichas personas y/o colectivo.

Pasando a los tweets educativos, hemos de destacar que el $41 \%$ de los publicados tenían esa característica según las determinaciones antes mencionadas en este estudio. Podemos dividirlos en dos grandes grupos: por un lado, aquellos que 
reivindican una mejora educativa sustancial para la etnia gitana, sobre todo con la crítica hacia la segregación en centros escolares de enseñanza obligatoria. Por otro, la exaltación de personas gitanas que han acabados estudios superiores de carácter no obligatorio, mayormente universitarios, quienes son vistos como referentes dentro de la propia comunidad.

No obstante, este sentimiento expresado a través de las redes sociales por los usuarios necesita de puntualizaciones concretas para poder comprenderse. Según los datos aportados por la Agencia de los Derechos Fundamentales de la Unión Europea en España el 31\% de niños gitanos estaban en centros con una elevada tasa de alumnado de esa etnia, lo que impide la integración con el resto de la sociedad. Estos centros han sido denominados por algunos como guetos educativos (Goenechea, 2016; Labra, 2015). Concretamente, el número de centros que muestran una alta concentración de alumnado de etnia gitana ha sido cuantificado en 173 según la Fundación Secretariado Gitano (Campos, 2018), y que se suma a la estigmatización y discriminación que expresan también en redes sociales.

A pesar de esta situación, la visión y avance en la escolarización del alumnado de etnia gitana ha alcanzado cotas hasta ahora desconocidas, aunque es cierto que existen notables diferencias de género. $\mathrm{Su}$ evolución y progresión en la escolarización en Primaria pretende ser del $99 \%$ en 2020, similar al resto. Por otra parte, es notable la evolución en la alfabetización, tanto en aquellos con edad escolar, como en personas adultas (Gitano, 2013a). Aún así, y teniendo en cuenta la veracidad de las expresiones vertidas en Twitter sobre esa marginación en determinados centros escolares, existen otros elementos que no se mencionan en las redes y que son destacables como: el uso de las nuevas tecnologías como herramientas de inclusión (Cabero y Ruiz Palmero, 2018), las diferencias entre la vida familiar y las directrices del sistema educativo; poca experiencia y cualificación de los docentes; falta de recursos, poca visibilidad de su cultura, costumbres, etc., en los currículos educativos, o la visibilización en los mass-media de los proyectos y personalidades relevantes de la etnia gitana (Consejería de Igualdad y Políticas Sociales, 2017).

\section{CONCLUSIONES}

A lo largo de nuestro estudio hemos podido comprobar el impacto y uso de las redes sociales, concretamente Twitter, a través del análisis del hashtag \#DíaInternacionaldelPuebloGitano con el fin de conocer lo reflejado en dicha red sobre la educación de la etnia gitana en España.

Podemos determinar, por tanto, que el impacto en términos generales del hashtag ha supuesto un incremento en redes sociales sobre la etnia gitana.

Así pues, el análisis cualitativo nos deja entrever las variedades y características personales a la hora de publicar un tweet $\mathrm{y}$, además, su número de emisiones con carácter educativo. De ambas podemos concluir que existe una diferencia notable de criterios por los usuarios a la hora de expresar sus críticas negativas y/o despectivas en redes sociales, aunque no es así a través de otros canales de comunicación. Por otro lado, hemos detectado como son similares las peticiones realizadas por los colectivos encargados del estudio y análisis de la educación de la etnia gitana, a las publicadas en redes sociales por la mayoría de los usuarios, centradas en: acabar con la discriminación y la falta de integración real en la sociedad, la mejora de la calidad educativa y la supresión de los centros con altas ratios de alumnado gitano, la motivación a través de los casos de éxitos académicos de personas de etnia gitana, etc.

Así pues, se hace necesario alabar la función de los colectivos y entidades, al mismo tiempo que realizar autocríticas sobre la situación que nos ha traído hasta aquí, teniendo en cuenta el crecimiento en el número de escolarizados, alumnado gitano con titulación cada vez más elevada, reducción de las tasas de alfabetización, etc. Todo ello, sin olvidar las diferencias de género entre niñas y niños a la hora de concluir sus estudios.

Por todo ello, creemos necesario continuar las investigaciones en este campo con el fin de determinar los patrones de comportamiento y opinión en el resto de redes sociales para dibujar con mayor precisión la realidad educativa a través de estos canales de comunicación social.

\section{REFERENCIAS}

Adnan, M., Leak, A., y Longleya, P. (2014). A geocomputational analysis of Twitter activity around different World Cities. Geo-spatial Information Science, $17(3), 145-152$. https://doi.org/10.1080/10095020.2014.941316

Bennett, W.L., y Segerberg, A. (2013). The logic of connective action: Digital media and the personalization of contentious politics. Massachusetts: Cambridge University Press.

Cabero, J., y Ruiz Palmero, J. (2018). Las Tecnologías de la información y la comunicación para la inclusión: reformulando la brecha digital. International Journal of Educational Research and Innovation (IJERI), 9, 16-30.

Campos, P. (2018). Así se ven los gitanos: perseguidos, rechazados, excluidos y marginados. La voz de Asturias. 
Recuperado

de

https://www.lavozdeasturias.es/noticia/asturias/2018/04/ 07/ven-gitanos-perseguidos-rechazados-excluidosdiscriminados/00031523122447717217395.htm

Consejería de Igualdad y Políticas Sociales (2017). Plan Integral para la Inclusión de la Comunidad Gitana de Andalucía 2017-2020. Junta de Andalucía.

Davis, K. (2015). Teachers' perceptions of Twitter for professional development. Disability and Rehabilitation, 37(17), 1551-1558.

De-Paoli, S., y Larooy, A. (2015). Teaching with Twitter: reflections on practices, opportunities and problems. En EUNIS 2015, Dundee, Scotland, 10-12 June.

European Union Agency for Fundamental Rights (2017). Second European Union Minorities and Discrimination Survey - Main results. Luxembourg. Publications Office of the European Union.

Garmendia, M., Garitaonandia, C., Martínez, G., y Casado, M. Á. (2011). Riesgos y seguridad en internet: Los menores españoles en el contexto europeo. Bilbao: Servicio Editorial de la Universidad del País Vasco.

Garreta i Bochaca, J. (1998). Escuela y minorías. Inmigrantes y gitanos ante el modelo educativo español. Revista de Educación, 317, 229-254.

Goenechea, C. (2016). La escuela pública de la España Multicultural: de privilegios y muros invisibles. Revista Interuniversitaria de Formación del Profesorado, 30(1), 111-119.

Gitano, F. S. (2013a). Estrategia Nacional Para la Inclusión Social de la Población Gitana en España 2012-2020. Fundación Secretariado Gitano, Ministro de Sanidad, Servicios Sociales e Igualdad, Madrid, España.

Gitano, F. S. (2013b). El alumnado gitano en Secundaria. Un estudio comparado. Ministerio de Educación, Cultura y Deporte.

Gitano, F. S. (2017). Informe anual FSG 2017. Discriminación y Comunidad Gitana. A.I.D., Madrid.

Iab Studio (2017). Estudio anual de Redes Sociales en España 2017. Elogia. Recuperado de https://marketing4ecommerce.net/viii-estudio-anual-deredes-sociales-en-espana-2017/

Jackson, L., y Wang, J.L. (2013). Cultural differences in social networking site use: a Comparative study of China and the United States. Computers in Human Behavior, 29, 910-921. https://doi.org/10.1016/j.chb.2012.11.024

Labra, J. P. (2015). Políticas educativas neoliberales para la integración de las TIC en educación. El caso de Madrid. Campus Virtuales, 1(1), 11-20.

Moreno, J. L. M., Navas, J. J. L., y Ortega, J. M. M. (2017). Protestando en Twitter: ciudadanía y empoderamiento desde la educación pública. Comunicar: Revista científica iberoamericana de comunicación y educación, (53), 39-48.

Padilla Carmona, M.T., González Monteagudo, J., y Soria Vílchez, A. (2017). Gitanos en la Universidad: Un estudio de caso de trayectorias de éxito en la Universidad de Sevilla. Revista de Educación, 377, 187211.

Rifkin, J. (2011). La Tercera Revolución Industrial: Cómo el poder lateral está transformando la energía, la economía y el mundo. Paidós.

Ruiz Palmero, J., Sánchez-Rodríguez, J. y Trujillo-Torres, J. M. (2016). Utilización de Internet y dependencia a teléfonos móviles en adolescentes. Revista Latinoamericana de Ciencias Sociales, Niñez y Juventud, 14(2), 1357-1369.

Sánchez Rodríguez, J., Ruiz Palmero, J., y Sánchez Rivas, E. (2015). Uso problemático de las redes sociales en estudiantes universitarios. Revista Complutense de Educación, 26, 159-174.

Sarlan, A., Nadam, C., y Basri, S. (2014). Twitter sentiment analysis. En Information Technology and Multimedia (ICIMU), 2014 International Conference, Putrajaya, Malaysia, 212-216.

Schwab, K. (2016). La cuarta revolución industrial. Debate.

Valdiviezo, J. L. C., Viteri, J. C. R., \& Carrera, G. M. D. (2017). Twitter en la Educación. Revista Publicando, 3(9), 238249.

Tur, G., Marín, V. I., \& Carpenter, J. (2017). Uso de Twitter en Educación Superior en España y Estados Unidos. Comunicar, 25(51), 19-28. (https://doi.org/10.3916/C512017-02)

Vera, G., y Dennys, N. (2017). Las redes sociales en el comportamiento de los adolescentes. Tesis Doctoral. Universidad de Guayaquil. Facultad de Filosofía, Letras y Ciencias de la Educación.

West, B., Moore, H., y Barry, B. (2015). Beyond the tweet: using Twitter to enhance engagement, learning, and success among First-year students. Journal of Marketing Education, $\quad 37(3)$, https://doi.org/10.1177/0273475315586061 